\title{
MicroRNA-224 Promotes Pancreatic Cancer Cell Proliferation and Migration by Targeting the TXNIP-Mediated HIF1 $\alpha$ Pathway
}

\author{
Guanghui Zhua,b,c Lianming Zhou ${ }^{a}$ Haijun Liua Yuanzhou Shan ${ }^{a}$ Xueli Zhang ${ }^{a, b, c}$ \\ ${ }^{a}$ Department of General Surgery, Shanghai Fengxian District Central Hospital, Shanghai, bepartment \\ of General Surgery, Fengxian Hospital affiliated to Southern Medical University, Shanghai, 'East \\ China Normal University and Shanghai Fengxian District Central Hospital Joint Research Center for \\ Translational Medicine, Shanghai, China
}

\section{Key Words}

Mir-224 • Pancreatic ductal adenocarcinoma • TXNIP • HIF1 $\alpha$

\begin{abstract}
Background/Aims: MicroRNAs (miRNAs) have been shown to participate in the development of pancreatic ductal adenocarcinoma (PDAC) by modulating multiple cellular processes. Increased miR-224 expression enhances proliferation and metastasis in human cancers. This study aimed to investigate the role of miR-224 and its underlying mechanism of action in PDAC. Methods: BrdU, MTT, and cell migration assays were performed to determine cell proliferation, viability, and migration, respectively. The binding sites of miR-224 were identified using a luciferase reporter system, whereas protein expression of target genes was determined by immunoblotting and immunofluorescence analyses. A BALB/C nude mouse xenograft model was used to evaluate the role of miR-224 in vivo. Results: We demonstrated that miR-224 expression was enhanced in PDAC cells and tissues, and was related to migration and proliferation. Noticeably, miR-224 overexpression promoted the proliferation, migration, and metastasis of Panc1 cells, while miR-224 inhibition had the reverse effect on PDAC cells. Moreover, we found that thioredoxin-interacting protein (TXNIP) is a target of miR-224. The results also indicated that miR-224 inversely regulated TXNIP by binding directly to its 3 '-untranslated region, which resulted in the activation of hypoxia-inducible factor $1 \alpha$ (HIF1 $\alpha$ ). Further, either TXNIP re-expression or HIF1 $\alpha$ depletion abolished the effects of miR-224 on the proliferation and migration of PDAC cells in vitro and in vivo. Regarding the relationship of TXNIP and HIF1 $\alpha$, we found that TXNIP mediated the nuclear export of HIF1 $\alpha$ and its degradation by forming a complex with HIF1 $\alpha$. Conclusion: The miR-224-TXNIP-HIF1 $\alpha$ axis may be useful in developing novel therapies for PDAC.




\section{Introduction}

Pancreatic ductal adenocarcinoma (PDAC) is a major contributor to cancer-specific mortality, which is catastrophic due to the limited number of treatment options [1, 2]. Although survival has been improved remarkably in patients with PDAC due to the development of therapeutic technologies, its clinical outcome is still unsatisfactory [2]. Contemporary treatments are insufficient for improving clinical outcome [3, 4]. Patients with PDAC usually have no symptoms and present with distant metastasis in organs, such as the lung or liver, or metastasis in proximal organs, including the stomach, intestine, and spleen [5]. Sometimes PDAC can only be identified when the malignancy has progressed to stage IV, subsequent to its surgical excision. Nevertheless, our understanding of relapse and metastasis in PDAC is still insufficient. Hence, the discovery of novel genes linked to metastasis and a better understanding of metastasis are crucial to the treatment of PDAC.

Until now, a large number of microRNAs (miRNAs) have been shown to be potentially effective in the treatment of malignancies [6-9]. MiRNAs are a group of endogenous evolutionarily conserved small non-coding RNAs, which have been proven to be essential in the development of PDAC, including cell proliferation, cell death, differentiation, angiogenesis, migration, metabolism, and invasion $[6,10,11]$. Recently, miR-224 was demonstrated to be upregulated in cervical cancer $[12,13]$. MiR-224 was previously described to be involved in the generation of tumors in hepatocellular carcinoma [14], and contributes to tumor migration, invasion, and cancer cell proliferation [14,15]. Moreover, miR-224 is upregulated in colorectal cancer, based on miRNA microarray data [16]. These studies suggest that miR224 acts as an oncogenic miRNA. However, the biological roles of miR-224 in PDAC remain unknown.

In this study, we aimed to explore the role of miR-224 and its underlying mechanism of action in PDAC. We found that miR-224 expression was upregulated in PDAC and significantly promoted cell proliferation and migration. From a mechanistic point of view, we also demonstrated that miR-224 inhibited the expression of thioredoxin-interacting protein (TXNIP) by targeting its 3'-untranslated region (UTR). Moreover, the downregulation of TXNIP led to the nuclear translocation of hypoxia-inducible factor $1 \alpha(\mathrm{HIF} 1 \alpha)$, an important regulator of growth in PDAC. Our findings provide a new therapeutic target for pancreatic cancer.

\section{Materials and Methods}

\section{Cell culture}

The Panc1, Miapaca2, AsPc-1, Panc02, and CFPAC1 human PDAC cell lines, and hTERT-HPNE normal pancreatic cells, were obtained from the American Type Culture Collection (Manassas, VA, USA). Another normal human pancreatic cell line, H6c7, was obtained from Kerafast (Boston, MA, USA). The cells were cultured in Dulbecco's modified Eagle's medium (Sigma, St. Louis, MO, USA) at $37^{\circ} \mathrm{C}$ under $5 \% \mathrm{CO}_{2}$.

\section{Tissue specimens}

A total of 49 pairs of human PDAC tissues and their corresponding matched adjacent non-tumor specimens were collected from the Shanghai Fengxian District Central Hospital, China. For the use of these tissues for research purposes, prior consent was obtained from the patients. Patient anonymity was also preserved.

Cell transfection

A miR-224 mimic, miR-224 inhibitor, pcDNA3.1-TXNIP, and small interfering RNAs (siRNAs) targeting TXNIP and HIF1 $\alpha$ were acquired from GenePharma (Shanghai, China). Two different siRNAs were used for TXNIP and HIF1 $\alpha$, respectively. The pGL4.20-HIF1 $\alpha$ plasmid was purchased from Addgene (Cambridge, MA, USA). The mutation in the TXNIP chromosomal maintenance 1 (CRM1) site (L294A) was generated using pcDNA3.1-TXNIP by site-directed mutagenesis (Thermo Fisher, Waltham, MA, USA). 


\section{Construction of stable miR-224 and TXNIP overexpressing cell lines}

To establish an miR-224 or miR-224 inhibitor overexpressing cell line, the pLV3-224-GFP or pLV3224i-GFP lentivirus expressing miR-224 or miR-224 anti-oligonucleotides were obtained from GenePharma. Panc1 cells were transduced with pLV3-224-GFP, which we refer to as Panc1-miR-224 cells. AsPc-1 cells were transduced with pLV3-224i-GFP, which were named AsPc-1-miR-224i. The efficiency of miR-224 or miR224 inhibitor overexpression was detected by quantitative real-time reverse transcription PCR (qRT-PCR). In order to establish the TXNIP stable overexpression cell lines, Panc1 or Panc1 miR-224 overexpressing cells were transfected with pcDNA3.1-TXNIP and subjected to neomycin screening. Stable overexpressing Panc1 cells were further referred to as Panc1-TXNIP and Panc1-miR-224-TXNIP.

\section{Western blotting and immunofluorescence}

Cell lysis was performed using RIPA buffer, and protein concentration was measured using the Bradford assay (Bio-Rad, Hercules, CA, USA). Identical amounts of samples were separated by 10-15\% sodium dodecyl sulfate-polyacrylamide gel electrophoresis. Proteins were detected with specific antibodies against E-cadherin (\#3195; Cell Signaling, BD), vimentin (SC-6260, V9; Santa Cruz), Ki-67 (ab15580; Abcam), TXNIP (K0205-3; MBL), HIF1 $\alpha$ (20960-1-AP; ProteinTech), and actin (A2228, Sigma) and the corresponding secondary antibodies. For immunofluorescence, the cells were fixed with paraformaldehyde, permeabilized, and incubated with the primary antibody overnight.

BrdU assay

Cell proliferation was detected using the BrdU incorporation assay (Roche Diagnostics GmbH, Mannheim, Germany). Absorbance was detected at $450 \mathrm{~nm}$.

\section{MTT assay}

MTT (3-(4, 5-dimethylthiazol-2-y1)-2, 5-diphenyltetrazolium bromide) assay was performed to measure cell viability. After treatment, MTT (10 mL, $5 \mathrm{mg} / \mathrm{mL})$ was added for $2-4 \mathrm{~h}$. After removing the culture medium, $75 \mathrm{~mL}$ dimethyl sulfoxide was added and incubated in the dark for $2 \mathrm{~h}$. Then, absorbance was measured at $490 \mathrm{~nm}$.

\section{Cell migration}

Cell migration was detected using the Transwell system (Corning, Inc., Corning, NY, USA). The cells (2.0 $\times 104$ ) were transfected, trypsinized, and seeded into the upper chamber. After incubation for $24 \mathrm{~h}$ at $37^{\circ} \mathrm{C}$, the cells that had migrated into the other compartment were fixed with methanol, stained with a $5 \%$ crystal violet solution, and counted. Each experiment was conducted with at least 3 replicates, and migrated cells were counted in more than 5 fields in each experiment.

\section{Luciferase assay}

The TXNIP 3'-UTR luciferase reporter was transfected into Panc1 cells expressing miR-224, or cotransfected with the miR-224 inhibitor into AsPc-1 cells. Luciferase activity was detected using a dual luciferase (Renilla and firefly) reporter assay system (Promega).

\section{In vivo experiments}

BALB/c nude mice (4-6 weeks old) were obtained from the Animal Center of the Chinese Academy of Sciences in Shanghai. The mice were randomized into 4 groups ( $\mathrm{n}=5$ ). Next, $1.0 \times 106$ Panc1-miR-224, Panc1-miR-NC, Panc1-TXNIP, Panc1-miR-224-TXNIP, AsPc-1-miR-224i, or AsPc-1-miR-NC cells were injected, respectively, into the tail vein to establish a metastatic mouse model. To assess the development of metastatic foci in the lung, the animals were sacrificed at 10 weeks after injection and hematoxylin and eosin (H\&E) staining was performed. The animal experiments were approved by the Ethics Review Committee of Shanghai Fengxian District Central Hospital.

\section{Statistical analysis}

Data are shown as the mean \pm standard deviation $(n=3)$. GraphPad Prism 6.0 (GraphPad, San Diego, CA, USA) was used to perform a two-tailed Student's t-test with Pearson's correlation analysis. $\mathrm{P}<0.05$ was considered statistically significant.

\section{KARGER}




\section{Results}

\section{MiR-224 is upregulated in pancreatic cancer clinical tissues and cells}

To investigate the role of miR-224 in the development of PDAC, we detected miR-224 expression levels by qRT-PCR. MiR-224 levels were significantly higher in pancreatic cancer tissues than in adjacent tissues $(\mathrm{P}<0.001)$ (Fig. 1A). We also found that miR-224 was clearly upregulated in aggressive PDAC tissues compared to non-aggressive tissues $(\mathrm{P}<0.01$, Fig. 1B). Moreover, we tested the expression levels of miR-224 in various PDAC cell lines and compared them with those observed in the H6c7 and hTERT-HPNE non-tumorigenic epithelial cell lines. Consistently, the levels of miR-224 were significantly increased in all five PDAC cell lines tested compared to the non-tumorigenic epithelial cell lines (Fig. 1C). Taken together, these data indicate that miR-224 upregulation is a common event in pancreatic cancer tissues. Thus, we propose that miR-224 might be involved in the progression of pancreatic tumors.

\section{MiR-224 stimulates PDAC cancer cell proliferation and migration}

To explore further the function of miR-224 in PDAC, miR-224 mimetic (miR) or inhibitor (miRi) was transfected in Panc1 and AsPc-1 cells, which express miR-224 at different levels (Fig. 1C). Firstly, we tested cell growth and proliferation in these cell lines using MTT and BrdU assays. MiR-224 transfection significantly increased the mRNA level of miR-224 (Fig. $\mathrm{S} 1 \mathrm{~A})$ and subsequently enhanced cell proliferation in a time-dependent manner in Panc1 cells (Fig. 2A\&B). For all supplemental material see www.karger.com/doi/10.1159/000492309. In contrast, inhibition of miR-224 by miRi suppressed the expression of miR-224 (Fig. S1B), and blocked the proliferation of AsPc-1 cells (Fig. 2C\&D). Since epithelial-to-mesenchymal transition has been recognized as an important regulator of cancer metastasis [17], we investigated whether miR-224 contributes to metastasis in PDAC via the regulation of epithelial-to-mesenchymal transition. We found that miR-224 overexpression promoted the migration of Panc1 cells (Fig. 2E), whereas miR-224 inhibition by miRi clearly blocked the migration of AsPc-1 cells (Fig. 2F). Moreover, our western blot analysis demonstrated that miR-224 overexpression suppressed the expression of the epithelial marker E-cadherin, but enhanced the levels of the mesenchymal marker vimentin and the proliferation marker Ki-67 in Panc1 cells (Fig. 2G). In contrast, loss of miR-224 had the reverse effect on the expression of Ki-67, E-cadherin, and vimentin in AsPc-1 cells (Fig. 2G). To confirm further the biological function of miR-224 in pancreatic cancer cell metastasis in vivo, AsPc-1 cells stably overexpressing miR-224 inhibitor (Panc1-miR-224i) and control cells (AsPc-1-miR-NC) were injected into the lateral vein of mice. Our results demonstrated that inhibition of miR-224 suppressed pancreatic cancer metastasis to the lung (Fig. 2H). These findings indicate that miR-224 stimulates PDAC growth and migration.

TXNIP is a direct target of miR-224 in PDAC cells

Previous studies have found that the oncogenic activity of miR-224 is exerted by targeting the $3^{\prime}$-UTR of TXNIP, which suppresses its expression [18]. Here, we

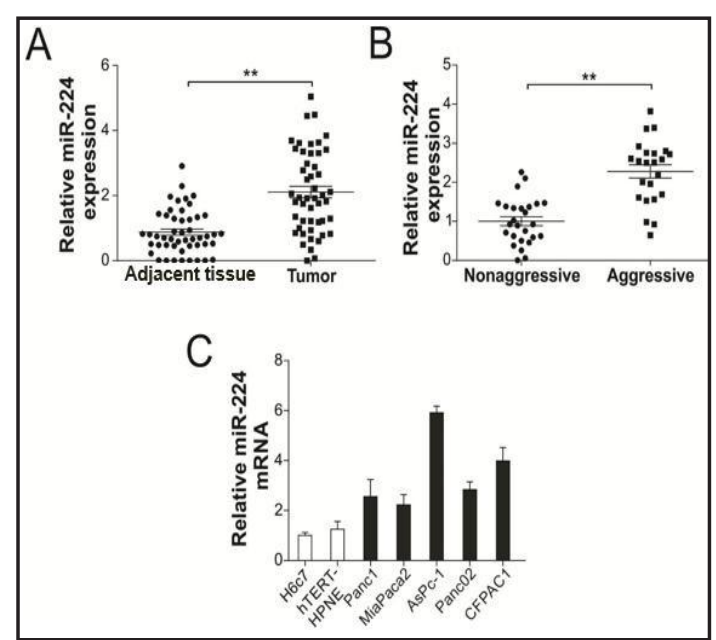

Fig. 1. MiR-224 expression is increased in PDAC. Comparison of the differences in miR-224 expression between (A) PDAC and matched tumoradjacent tissues; (B) aggressive and nonaggressive tumor tissues; and (C) PDAC cell lines and the immortalized pancreatic cell lines H6c7 and hTERTHPNE. ${ }^{* *} \mathrm{P}<0.01$. 
Fig. 2. MiR-224 inhibits the proliferation and migration of PDAC cells. (A) Panc1 cells that were transfected with miRNA-224 mimetic (miR) were subjected to MTT (left) and BrdU (right) assays at the indicated time points for cell proliferation. (B) Panc1 cells were treated as in (A) for $48 \mathrm{~h}$, and cell proliferation was determined by crystal violet staining. (C) AsPc-1 cells that were transfected with miRNA-224 inhibitor (miRi) were subjected to MTT (left) and BrdU (right) assays at the indicated time points for cell proliferation. (D) AsPc-1 cells were treated as in (C) for $48 \mathrm{~h}$, and cell proliferation was determined by crystal violet staining. (E) Panc1 cells were treated as in (A), and migration was measured by Transwell assays. There are at least 3 replicates for each experiment, and more than 5 fields of migrated cells were counted in each group. The same thereafter for the cell migration assay. (F) AsPc-1 cells were treated as in (C), and migration as measured by Transwell assays. (G) Panc1 (left) and AsPc-1 (right) cells that were transfected with miR or miRi, respectively, and the expression of the indicated proteins was analyzed by western blotting. (H) AsPc-1-miR-224i or AsPc-1-miR-NC cells $\left(1.0 \times 10^{6}\right.$ cells $)$ were injected into the tail vein of mice. Animals were sacrificed at 10 weeks after injection, and the development of metastatic foci in the lung was analyzed by $\mathrm{H} \& \mathrm{E}$ staining followed by histological analysis. ${ }^{* *} \mathrm{P}<0.01$.

discovered that both the mRNA and protein expression levels of TXNIP were significantly lower in all five PC cell lines tested than in normal pancreatic cells (Fig. 3A). This is in contrast to the expression of miR-224 in these cell lines. Furthermore, the higher expression of miRNA-224 in PDAC cells, such as AsPc-1 and CFAC1, correlated with the lower expression of TXNIP (Fig. 3A). Notably, an evident inverse correlation was found between the expression levels of miR-224 and TXNIP mRNA in PDAC tissues (P $<0.001$, Fig. 3B), suggesting that TXNIP might be a target of miR-224 in PDAC. To confirm this result, we further determined TXNIP expression in various PDAC cells treated with miR or miRi. Accordingly, TXNIP was also downregulated by miR-224 under these conditions, but its expression increased when miR-224 was inhibited (Fig. 3C\&D). Next, miR-224 overexpression was found to decrease luciferase activity, while luciferase activity was unchanged when the $3^{\prime}$-UTR sequences in the sites complementary to the seed region of miR-224 were mutated (Fig. 3E). Further, miRi significantly increased luciferase activity when the constructs were co-transfected into cells. However, luciferase activity did not change when the 3'-UTR sequences in TXNIP, in the seed region of miR-224, were mutated (Fig. 3E). These data show that TXNIP is the target of miR224 in PDAC cells.

TXNIP mediates the effects of miR-224 in vitro and in vivo

Since TXNIP is a target of miR-224 in the progression of PDAC, we further speculated that this oncogenic behavior is dependent of the inhibition of TXNIP. The depletion of TXNIP using two different siRNAs suppressed the expression E-cadherin, but augmented the expression of vimentin and Ki-67 in Panc1 cells (Fig. 4A and Fig. S2A). Accordingly, the silencing of TXNIP promoted cell proliferation and migration in Panc-1 cells (Fig. 4B\&C and Fig. S2B\&C), which had similar effects with miR-224 overexpression. Furthermore, the co-

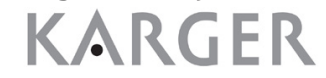


transfection of TXNIP with miR-224 mimics reversed the expression of Ki-67, E-cadherin, and vimentin (Fig. 4D), as well as the increase in growth induced by miR-224 (Fig. 4E\&F).

To confirm further the biological function of miR-224 and its relationship with TXNIP

Fig. 3. TXNIP is a target of miR-224 in PDAC cells. (A) The mRNA (upper) and protein (lower) expression of TXNIP in the indicated cell lines was tested by qRT-PCR and western blot analyses. The mRNA level of TXNIP was normalized to that in normal pancreatic cells (H6c7 cells). (B) The correlation between TXNIP and miR-224 in PDAC clinical samples was analyzed by Spearman's rank correlation $(\mathrm{P}=0.0011)$. (C) Panc1 and Miapaca2 cells were transfected with $\mathrm{miR}$, and TXNIP expression was analyzed by western blotting. (D) AsPc-1 and CFPAC1 cells were transfected with miRi, and TXNIP expression was analyzed by western blotting. (E) TXNIP 3'-UTR WT or miR224 binding site mutant (MT) luciferase activity in Panc1 cells transfected with miR. (F) TXNIP 3'-UTR WT or miR-224 binding site mutant (MT)

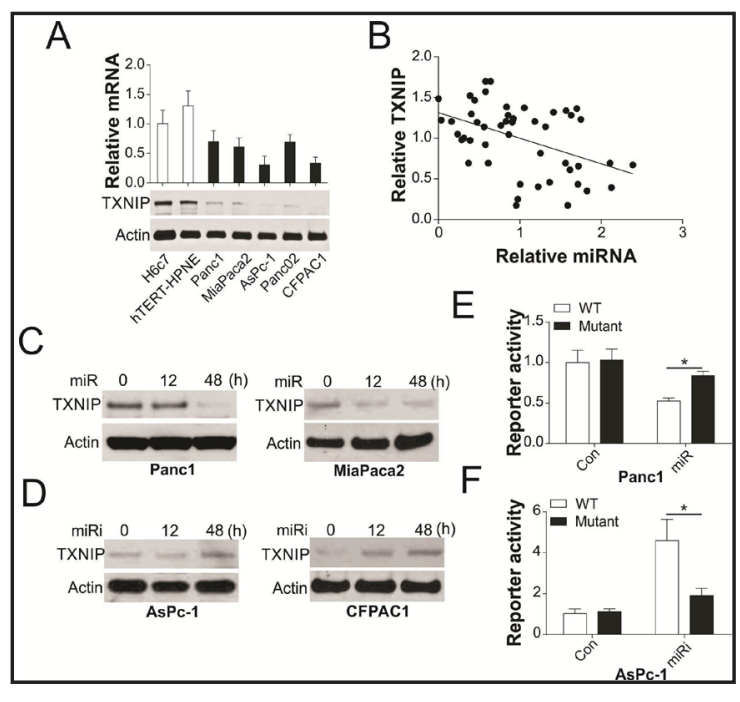
luciferase activity in AsPc-1 cells transfected with miRi. ${ }^{*} \mathrm{P}<0.05$.

Fig. 4. miR-224 promotes cell proliferation via TXNIP downregulation in PDAC cells. (A) Panc1 cells were transfected with TXNIP siRNA, and the expression of the indicated proteins was analyzed by western blotting. (B) The proliferation of Panc1 cells treated as in (A) was tested by an MTT assay (left), BrdU assay (middle), and crystal violet staining (right) at 48 h. (C) The migration of Panc1 cells treated as in (A) was tested by Transwell assays. (D) Panc1 cells were co-transfected with miR and TXNIP plasmid, and the expression of the indicated proteins was analyzed by western blotting. (E) The proliferation of Panc1 cells treated as in (D) was tested by MTT (left) and BrdU (right) assays at $48 \mathrm{~h}$. (F) The migration of Panc1 cells treated as in (D) was tested by Transwell assays. (G) Histological analysis of the pulmonary metastases in the animal model as described. ${ }^{*} \mathrm{P}<0.05$; ${ }^{* *} \mathrm{P}<0.01$.

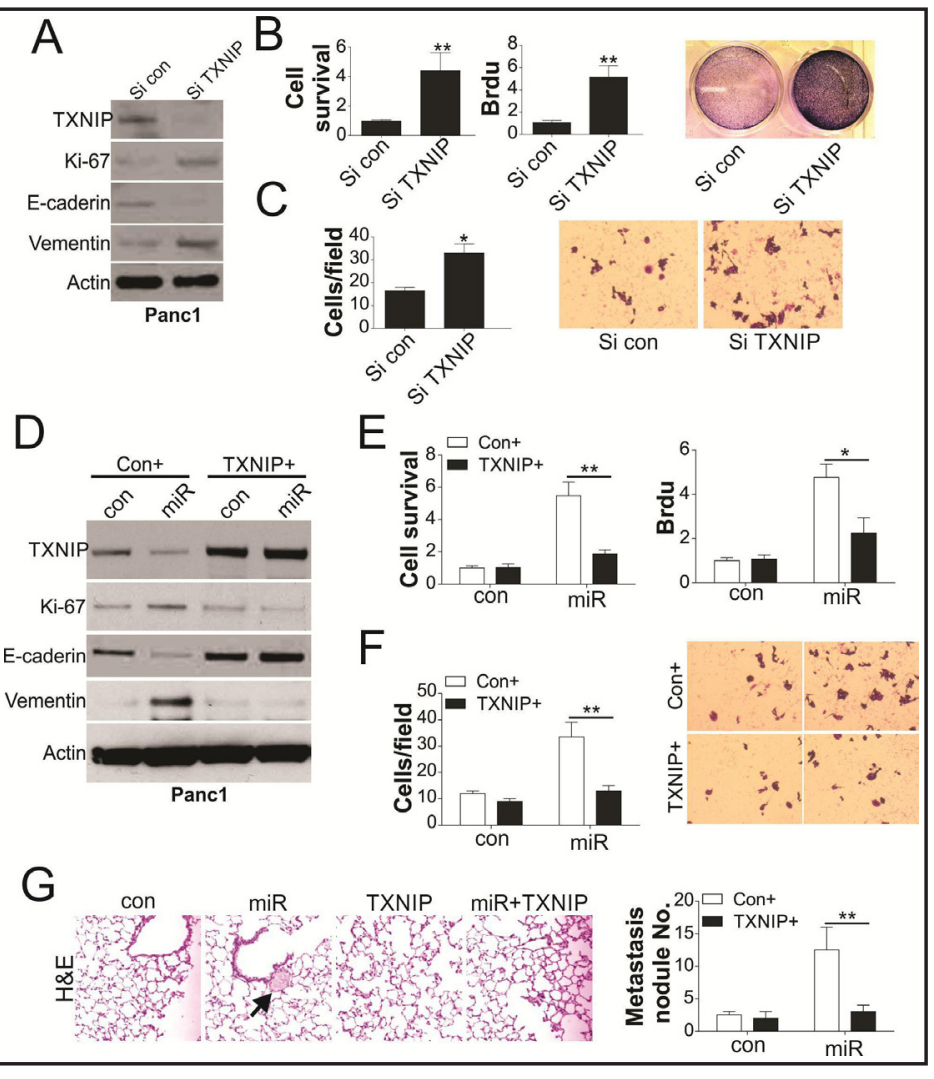


in vivo, Panc1 cells stably overexpressing miR-224 (Panc1-miR), TXNIP (Panc1-TXNIP), or co-expressing miR-224 and TXNIP (Panc1-miR+TXNIP), and control cells (Panc-miRNC) were injected into the lateral vein of mice. Our results demonstrated that the miR-224 overexpression group displayed more metastasis, observed as lung nodules, when examined microscopically (Fig. 4G). However, the co-expression of TXNIP and miR-224 resulted in a reduction in the number of lung nodules (Fig. 4G). Taken together, our data demonstrate that miR-224 leads to PDAC growth and migration by targeting and suppressing TXNIP.

\section{MiR-224 upregulates HIF1 $\alpha$ via the TXNIP pathway}

HIF1 $\alpha$ is a crucial factor in the induction of tumor proliferation, migration, and metastasis [19], and it is degraded by the von Hippel-Lindau protein (pVHL) under normoxia [20]. Here, we also demonstrated that the depletion of TXNIP by siRNA increased the expression of HIF1 $\alpha$ in Panc1 cells (Fig. 5A). In contrast, the enhanced expression of TXNIP compromised the induction of HIF1 $\alpha$ following miR-224 transfection (Fig. 5B). However, no significant changes in HIF1 $\alpha$ mRNA levels were observed upon TXNIP knockdown or miR-224 transfection (Fig. 5C), suggesting that TXNIP and miR-224 modulate HIF1 $\alpha$ at the protein level. Accordingly, the downregulation of HIF1 $\alpha$ using an miR-224 inhibitor could be rescued by pretreatment with the protease inhibitor MG132 (Fig. 5D). Previous studies have indicated that TXNIP is associated with the $\beta$-domain of pVHL and promotes the degradation of HIF1 $\alpha$ independently of hypoxia [20]. We therefore tested the interaction

Fig. 5. MiR-224 suppresses the expression of TXNIP to activate HIF1 $\alpha$. (A) Panc1 cells were transfected with TXNIP siRNA, and HIF1 $\alpha$ expression was analyzed by western blotting. (B) Panc1 cells were co-transfected with miR and TXNIP plasmid, and HIF1 $\alpha$ expression was analyzed by western blotting. (C) The expression of HIF1 $\alpha$ mRNA in Panc1 cells transfected with TXNIP siRNA or miR. (D) AsPc-1 cells were pretreated with $5 \mu \mathrm{M}$ MG132 for $2 \mathrm{~h}$, then transfected with miRi for another $6 \mathrm{~h}$, and the expression of HIF1 $\alpha$ was analyzed by western blotting. (E) AsPc-1 cells were pretreated with MG132 for $2 \mathrm{~h}$ and then co-transfected with miRi and TXNIP siRNA as indicated. The interaction of TXNIP and HIF1 $\alpha$, and HIF1 $\alpha$ ubiquitination were tested by immunoprecipitation (IP) with an anti-HIF1 $\alpha$ antibody. (F) Panc1 cells were pretreated with MG132 for $2 \mathrm{~h}$ and then co-

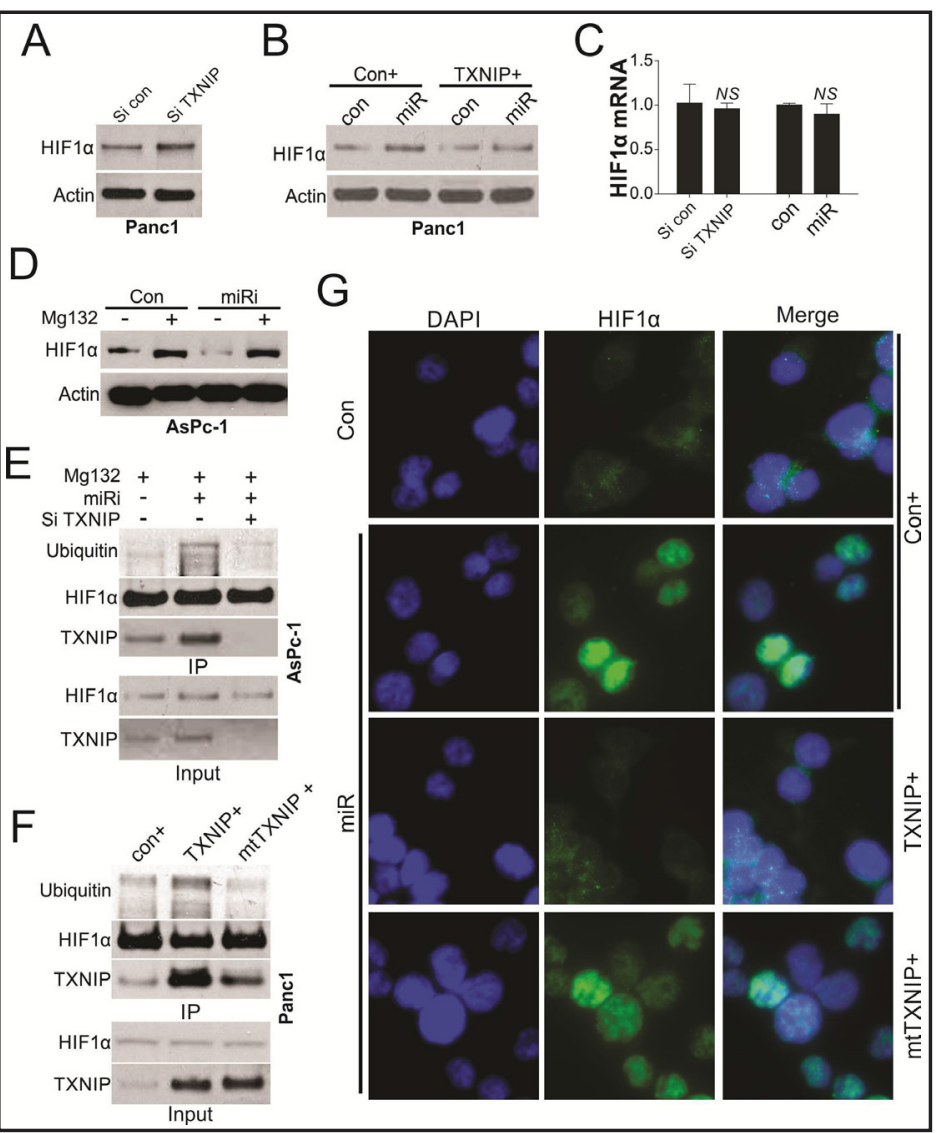
transfected with miR and TXNIP WT or CRM1 mutant plasmid (mtTXNIP) as indicated. At $6 \mathrm{~h}$ later, the interaction of TXNIP and HIF1 $\alpha$, and HIF1 $\alpha$ ubiquitination were tested by IP with an anti-HIF1 $\alpha$ antibody. (G) Panc1 cells were co-transfected with miR and TXNIP WT or mtTXNIP as indicated. The expression and cellular localization of HIF1 $\alpha$ was analyzed by immunofluorescence staining. Not significant (NS), P > 0.05.

\section{KARGER}


between TXNIP and HIF1 $\alpha$ and the ubiquitination status of HIF1 $\alpha$, and found that the miR224 inhibitor enhanced the interaction between TXNIP and HIF1 $\alpha$ and the ubiquitination of HIF1 $\alpha$ upon MG132 pretreatment (Fig. 5E). However, TXNIP knockdown abolished the ubiquitination of HIF $1 \alpha$, indicating that the interaction between TXNIP and HIF $1 \alpha$ promotes its degradation. As the CRM1 binding site (Leu294) in TXNIP is important for the formation of the TXNIP-pVHL-HIF1 $\alpha$ complex [21], we transfected AsPc-1 cells with the TXNIP construct or the TXNIP construct containing the CRM1 binding site mutation. We found that the enhanced expression of wild-type TXNIP increased the interaction with HIF1 $\alpha$ and HIF $1 \alpha$ ubiquitination, which was absent when mutant TXNIP was transfected (Fig. 5F). Furthermore, miR-224 transfection led to HIF1 $\alpha$ stabilization and translocation into the nucleus, which was abolished by co-transfection with wild-type TXNIP, but not mutant TXNIP (Fig. 5G). These findings suggest that miR-224 suppresses TXNIP expression to release and stabilize HIF $1 \alpha$.

\section{HIF1 $\alpha$ is necessary for miR-224-mediated growth}

Finally, we determined whether HIF1 $\alpha$ is necessary for miR-224-mediated cell proliferation and migration in PDAC. As predicted, miR-224 overexpression in Panc1 cells increased the expression of HIF1 $\alpha$, which was correlated with the augmentation of cell proliferation and migration (Fig. 6A-C and Fig. S3). In contrast, knockdown of HIF1 $\alpha$ compromised the growth induced by transfection with miR-224 (Fig. 6A-C and Fig. S3). Conversely, suppression of miR-224 by its inhibitor decreased HIF1 $\alpha$ expression, cell proliferation, and migration (Fig. 6D-F), which was reversed by the enhanced expression of HIF1 $\alpha$ (Fig. 6E-F). These results indicate that miR-224 increases HIF1 $\alpha$ levels and activity by downregulating TXNIP, which is essential for PDAC cell growth and migration.

\section{Discussion}

The distinct expression of miRNAs between malignant and normal specimens or among groups of malignant specimens with a favorable or unsatisfying prognosis has been used

Fig. 6. HIF $1 \alpha$ is essential for miR224 in PDAC cell proliferation and migration. (A) Panc1 cells were cotransfected with miR and HIF1 $\alpha$ siRNA, and the knockdown effect of HIF1 $\alpha$ was verified by western blotting. (B) The proliferation of Panc1 cells treated as in (A) was tested by MTT (left) and BrdU (right) assays at $48 \mathrm{~h}$. (C) The migration of Panc1 cells treated as in (A) was tested by Transwell assays. (D) AsPc-1 cells were cotransfected with miRi and HIF1 $\alpha$ plasmid, and HIF1 $\alpha$ expression was analyzed by western blotting. (E) The proliferation of Panc1 cells treated as in (D) was tested by MTT (left) and BrdU (right) assays at $48 \mathrm{~h}$. (F) The migration of Panc1 cells treated as in (D) was tested by Transwell assays. $* \mathrm{P}<0.05$; ${ }^{* *} \mathrm{P}<0.01$.

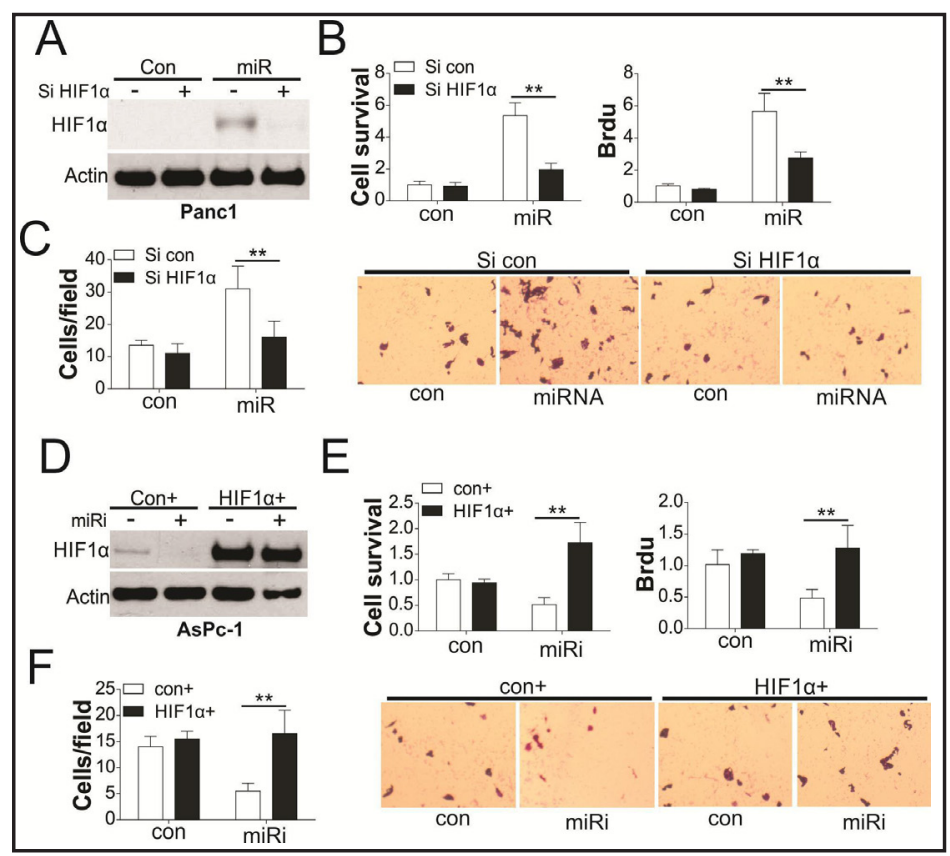


to obtain miRNA signatures with promising predictive and/or prognostic values [22, 23]. MiRNA profiling of PDAC tissues has identified several hundreds to thousands of miRNAs associated with tumor progression [6]. However, only a few studies have described the correlation between miR-224 and pancreatic cancer. Here, we showed that miR-224 expression was upregulated in aggressive tumor samples compared with nonaggressive tumor samples. Furthermore, our results demonstrated that miR-224 promoted PDAC cell proliferation and migration in vitro, as well as metastasis to the lung in vivo. Thus, miR-224 acts as an oncogene to promote the progression and metastasis of pancreatic cancer, which is consistent with previous studies [13,24].

Although miR-224 is overexpressed in various cancers and promotes cancer progression [25], the mechanism underlying the role of this miRNA in cancer is understood poorly. In this study, a novel mechanism underlying the role of miR-224 was elucidated, namely that miR224 promotes PDAC cell growth through miR-224-TXNIP-HIF1 $\alpha$ signaling. A previous report showed that E2F1 modulates the expression of miR-224 by targeting TXNIP, which can drive cancer metastasis in melanoma [18]. However, the mechanism through which TXNIP inhibits cancer progression was not addressed clearly. In this study, we have also shown that miR224 targets TXNIP and suppresses its expression in pancreatic cancer, which may promote PDAC growth. Furthermore, the suppression of TXNIP by miR-224 leads to the nuclear translocation of HIF1 $\alpha$, which is an important stimulator of cancer progression.

The loss of TXNIP has been shown to correlate with the advanced stage of breast, gastric, colorectal, and bladder cancers [20, 26, 27]. However, TXNIP genetic alterations, such as deletions, translocations, or somatic mutations, have not been found in these cancers [28]. In this study, we found that miR-224 targets TXNIP and leads to its suppression in PDAC. TXNIP has also been reported as a suppressor of metastasis $[18,29]$. Consistent with these findings, we observed that the enhanced expression of TXNIP reduced miR-224-induced cell growth and metastasis in PDAC. TXNIP was primarily recognized as a thioredoxin-binding protein that inhibits thioredoxin [30]. Since thioredoxin promotes tumor progression by inducing angiogenesis [31] and inhibiting apoptosis [32], TXNIP has great potential as a tumor suppressor gene. A previous study also found that miR-337 overexpression led to TXNIP silencing, and decreased the levels of intracellular reactive oxygen species (ROS), which activated HIF1 $\alpha$ and TWIST [33]. Nevertheless, recent research has revealed that TXNIP plays a crucial role in redox-independent signaling [21]. TXNIP was found to inhibit glucose uptake independent of thioredoxin binding, which was important for the survival of thyroid cancer cells [34]. Interestingly, several studies have also shown that the overexpression of TXNIP inhibited HIF-mediated reporter activity in various cancer cells [20, 33, 35]. Moreover, they found that the CRM1 region of TXNIP binds the nuclear transcription factor HIF1 $\alpha$ and the ubiquitin ligase pVHL. The TXNIP-pVHL-HIF1 $\alpha$ complex results in the degradation of HIF1 $\alpha$ [24]. In accordance with these results, our study also showed that TXNIP negatively regulated HIF1 $\alpha$, independently of its interaction with thioredoxin and ROS, as no obvious change in HIF1 $\alpha$ mRNA levels was observed. Instead, our results suggest that TXNIP can form a complex with HIF1 $\alpha$, which leads to HIF1 $\alpha$ degradation and inhibition of its nuclear translocation.

The modulation of HIF1 $\alpha$ concentration is essential to manage the expression of diverse genes involved in the hypoxic reaction, vessel generation, oxygen transport, and glucose metabolism, which promote migration, growth, and tumor invasion [36, 37]. In cells, HIF $1 \alpha$ is regulated through various pathways, such as transcription, translation, and posttranslational mechanisms, protein-protein interactions, and degradation [36, 37]. Besides hypoxia mimetics, various hypoxic miRNAs that affect HIF1 $\alpha$ expression have been identified $[38,39]$. Here, we showed that miR-224 elevated HIF1 $\alpha$ levels and activated HIF1 $\alpha$ by directly targeting TXNIP. The co-expression of TXNIP blocks the induction of growth and the upregulation of HIF1 $\alpha$ induced by miR-224. However, as opposed to previous studies, which showed that miR-373 activated HIF1 $\alpha$ by ROS scavenging, our results suggested that miR-224 induces HIF1 $\alpha$ expression by enhancing its nuclear translocation and stabilization. It has been shown that HIF1 $\alpha$ localization and degradation occurs in a cell type-dependent 
manner and varies in response to different stimuli [40, 41]. In transformed HeG2 cells, HIF1 $\alpha$ degradation mainly occurred in the cytoplasm, while in primary endothelial cells, it occurred in the nucleus and cytoplasm. Therefore, our data suggest that the miR-224-TXNIP-HIF1 $\alpha$ axis is important for PDAC cell proliferation and migration, which creates novel opportunities for cancer therapeutics.

In summary, our results suggest that miR-224 drives pancreatic cancer cell proliferation and migration. From a mechanistic perspective, miR-224 promotes cancer progression and metastasis via the miR-224-TXNIP-HIF1 $\alpha$ axis in PDAC. Components of this axis may be utilized as potential therapeutic targets and biomarkers for PDAC.

\section{Acknowledgements}

This study was supported by Natural Science Foundation of China (NO. 81402377).

\section{Disclosure Statement}

The authors declare no potential conflict of interests.

\section{References}

1 Matsumoto K, Takeda Y, Onoyama T, Kawata S, Kurumi H, Koda H, Yamashita T, Isomoto H: Endoscopic treatment for distal malignant biliary obstruction. Ann Transl Med 2017;5:190.

-2 Chrystoja CC, Diamandis EP, Brand R, Ruckert F, Haun R, Molina R: Pancreatic cancer. Clin Chem 2013;59:41-46.

-3 Bournet B, Buscail C, Muscari F, Cordelier P, Buscail L: Targeting KRAS for diagnosis, prognosis, and treatment of pancreatic cancer: Hopes and realities. Eur J Cancer 2016;54:75-83.

4 Deng T, Yuan Y, Zhang C, Zhang C, Yao W, Wang C, Liu R, Ba Y: Identification of circulating miR-25 as a potential biomarker for pancreatic cancer diagnosis. Cell Physiol Biochem 2016;39:1716-1722.

5 Jafarnejad SM, Li G: Regulation of p53 by ING family members in suppression of tumor initiation and progression. Cancer Metastasis Rev 2012;31:55-73.

6 Yonemori K, Kurahara H, Maemura K, Natsugoe S: MicroRNA in pancreatic cancer. J Hum Genet 2017;62:3340.

7 Zhou B, Sun C, Hu X, Zhan H, Zou H, Feng Y, Qiu F, Zhang S, Wu L, Zhang B: MicroRNA-195 Suppresses the Progression of Pancreatic Cancer by Targeting DCLK1 Cell Physio Biochem 2017;44:1867-1881.

-8 Qian Y, Feng L, Wu W, Weng T, Hu C, Hong B, Wang FX, Shen L, Wang Q, Jin X: MicroRNA Expression Profiling of Pancreatic Cancer Cell Line L36p1 Following B7-H4 Knockdown. Cell Physio Biochem 2017;44:494504.

-9 Yang W, Yang Y, Xia L, Yang Y, Wang F, Song M, Chen X, Liu J, Song Y, Zhao Y: MiR-221 promotes Capan-2 pancreatic ductal adenocarcinoma cells proliferation by targeting PTEN-Akt. Cell Physio Biochem 2016;38:2366-2374.

10 Zhao L, Sun H, Kong H, Chen Z, Chen B, Zhou M: The Lncrna-TUG1/EZH2 axis promotes pancreatic cancer cell proliferation, migration and EMT phenotype formation through sponging Mir-382. Cell Physio Biochem 2017;42:2145-2158.

11 Li H, Hao X, Wang H, Liu Z, He Y, Pu M, Zhang H, Yu H, Duan J, Qu S: Circular RNA expression profile of pancreatic ductal adenocarcinoma revealed by microarray. Cell Physio Biochem 2016;40:1334-1344.

12 Shen SN, Wang LF, Jia YF, Hao YQ, Zhang L, Wang H: Upregulation of microRNA-224 is associated with aggressive progression and poor prognosis in human cervical cancer. Diagn Pathol 2013;8:69.

13 Mees ST, Mardin WA, Sielker S, Willscher E, Senninger N, Schleicher C, Colombo-Benkmann M, Haier $\mathrm{J}$ : Involvement of CD40 targeting miR-224 and miR-486 on the progression of pancreatic ductal 


\section{Cellular Physiology Cell Physiol Biochem 2018;48:1735-1746

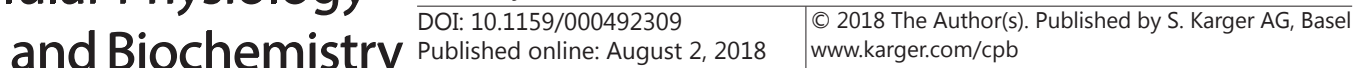

adenocarcinomas. Ann Surg Oncol 2009;16:2339-2350.

14 Zhang Y, Takahashi S, Tasaka A, Yoshima T, Ochi H, Chayama K: Involvement of microRNA-224 in cell proliferation, migration, invasion, and anti-apoptosis in hepatocellular carcinoma. J Gastroenterol Hepatol 2013;28:565-575.

15 Scisciani C, Vossio S, Guerrieri F, Schinzari V, De Iaco R, D’Onorio de Meo P, Cervello M, Montalto G, Pollicino T, Raimondo G, Levrero M, Pediconi N: Transcriptional regulation of miR-224 upregulated in human HCCs by NFkappaB inflammatory pathways. J Hepatol 2012;56:855-861.

16 Arndt GM, Dossey L, Cullen LM, Lai A, Druker R, Eisbacher M, Zhang C, Tran N, Fan H, Retzlaff K, Bittner A, Raponi M: Characterization of global microRNA expression reveals oncogenic potential of miR-145 in metastatic colorectal cancer. BMC Cancer 2009;9:374.

17 Jayachandran A, Dhungel B, Steel JC: Epithelial-to-mesenchymal plasticity of cancer stem cells: therapeutic targets in hepatocellular carcinoma. J Hematol Oncol 2016;9:74.

-18 Knoll S, Furst K, Kowtharapu B, Schmitz U, Marquardt S, Wolkenhauer O, Martin H, Putzer BM: E2F1 induces miR-224/452 expression to drive EMT through TXNIP downregulation. EMBO Rep 2014;15:13151329.

19 Schofield CJ, Ratcliffe PJ: Oxygen sensing by HIF hydroxylases. Nature reviews. Molecular cell biology 2004;5:343-354.

20 Shin D, Jeon JH, Jeong M, Suh HW, Kim S, Kim HC, Moon OS, Kim YS, Chung JW, Yoon SR, Kim WH, Choi I: VDUP1 mediates nuclear export of HIF1alpha via CRM1-dependent pathway. Biochimica et biophysica acta 2008;1783:838-848.

21 Spindel ON, World C, Berk BC: Thioredoxin interacting protein: redox dependent and independent regulatory mechanisms. Antioxid Redox Signal 2012;16:587-596.

-22 Garzon R, Marcucci G: Potential of microRNAs for cancer diagnostics, prognostication and therapy. Current opinion in oncology 2012;24:655-659.

23 Albulescu R, Neagu M, Albulescu L, Tanase C: Tissular and soluble miRNAs for diagnostic and therapy improvement in digestive tract cancers. Expert review of molecular diagnostics 2011;11:101-120.

24 Bai J, Na H, Hua X, Wei Y, Ye T, Zhang Y, Jian G, Zeng W, Yan L, Tang Q: A retrospective study of NENs and miR-224 promotes apoptosis of BON-1 cells by targeting PCSK9 inhibition. Oncotarget 2017;8:6929-6939.

25 He C, Wang L, Zhang J, Xu H: Hypoxia-inducible microRNA-224 promotes the cell growth, migration and invasion by directly targeting RASSF8 in gastric cancer. Molecular cancer 2017;16:35.

-26 Nishizawa K, Nishiyama H, Matsui Y, Kobayashi T, Saito R, Kotani H, Masutani H, Oishi S, Toda Y, Fujii N, Yodoi J, Ogawa O: Thioredoxin-interacting protein suppresses bladder carcinogenesis. Carcinogenesis 2011;32:1459-1466.

27 Woolston CM, Madhusudan S, Soomro IN, Lobo DN, Reece-Smith AM, Parsons SL, Martin SG: Thioredoxin interacting protein and its association with clinical outcome in gastro-oesophageal adenocarcinoma. Redox biology 2013;1:285-291.

-28 Zhou J, Yu Q, Chng WJ: TXNIP (VDUP-1, TBP-2): a major redox regulator commonly suppressed in cancer by epigenetic mechanisms. The international journal of biochemistry \& cell biology 2011;43:1668-1673.

-29 Goldberg SF, Miele ME, Hatta N, Takata M, Paquette-Straub C, Freedman LP, Welch DR: Melanoma metastasis suppression by chromosome 6: evidence for a pathway regulated by CRSP3 and TXNIP. Cancer research 2003;63:432-440.

-30 Nishiyama A, Matsui M, Iwata S, Hirota K, Masutani H, Nakamura H, Takagi Y, Sono H, Gon Y, Yodoi J: Identification of thioredoxin-binding protein-2/vitamin $\mathrm{D}(3)$ up-regulated protein 1 as a negative regulator of thioredoxin function and expression. J Biol Chem 1999;274:21645-21650.

31 Welsh SJ, Bellamy WT, Briehl MM, Powis G: The redox protein thioredoxin-1 (Trx-1) increases hypoxiainducible factor 1alpha protein expression: Trx-1 overexpression results in increased vascular endothelial growth factor production and enhanced tumor angiogenesis. Cancer Res 2002;62:5089-5095.

32 Saitoh M, Nishitoh H, Fujii M, Takeda K, Tobiume K, Sawada Y, Kawabata M, Miyazono K, Ichijo H: Mammalian thioredoxin is a direct inhibitor of apoptosis signal-regulating kinase (ASK) 1. EMBO J 1998;17:2596-2606.

-33 Chen D, Dang BL, Huang JZ, Chen M, Wu D, Xu ML, Li R, Yan GR: MiR-373 drives the epithelial-tomesenchymal transition and metastasis via the miR-373-TXNIP-HIF1alpha-TWIST signaling axis in breast 


\section{Cellular Physiology Cell Physiol Biochem 2018;48:1735-1746

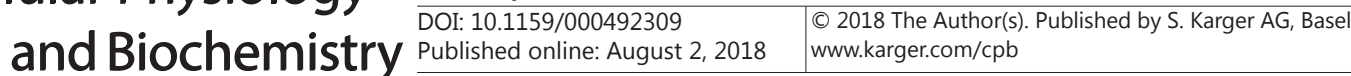 \\ Zhu et al.: Microrna-224 Promotes Pancreatic Cancer Cell}

cancer. Oncotarget 2015;6:32701-32712.

-34 Waldhart AN, Dykstra H, Peck AS, Boguslawski EA, Madaj ZB, Wen J, Veldkamp K, Hollowell M, Zheng B, Cantley LC, McGraw TE, Wu N: Phosphorylation of TXNIP by AKT Mediates Acute Influx of Glucose in Response to Insulin. Cell Rep 2017;19:2005-2013.

-35 Wong RW, Hagen T: Mechanistic target of rapamycin (mTOR) dependent regulation of thioredoxin interacting protein (TXNIP) transcription in hypoxia. Biochem Biophys Res Commun 2013;433:40-46.

-36 Yang MH, Wu MZ, Chiou SH, Chen PM, Chang SY, Liu CJ, Teng SC, Wu KJ: Direct regulation of TWIST by HIF1alpha promotes metastasis. Nat Cell Biol 2008;10:295-305.

-37 Gao P, Zhang H, Dinavahi R, Li F, Xiang Y, Raman V, Bhujwalla ZM, Felsher DW, Cheng L, Pevsner J, Lee LA, Semenza GL, Dang CV: HIF-dependent antitumorigenic effect of antioxidants in vivo. Cancer Cell 2007;12:230-238.

38 Madanecki P, Kapoor N, Bebok Z, Ochocka R, Collawn JF, Bartoszewski R: Regulation of angiogenesis by hypoxia: the role of microRNA. Cell Mol Biol Lett 2013;18:47-57.

39 Greco S, Martelli F: MicroRNAs in Hypoxia Response. Antioxid Redox Signal 2014;21:1164-1166.

40 Carbajo-Pescador S, Ordonez R, Benet M, Jover R, Garcia-Palomo A, Mauriz JL, Gonzalez-Gallego J: Inhibition of VEGF expression through blockade of Hif1alpha and STAT3 signalling mediates the antiangiogenic effect of melatonin in HepG2 liver cancer cells. Br J Cancer 2013;109:83-91.

41 Sanagawa A, Iwaki S, Asai M, Sakakibara D, Norimoto H, Sobel BE, Fujii S: Sphingosine 1phosphate induced by hypoxia increases the expression of PAI1 in HepG2 cells via HIF1alpha. Mol Med Rep 2016;14:18411848. 